\title{
Compositional Dependence of the Formation Energies of Substitutional and Interstitial Mn in Partially Compensated (Ga,Mn)As
}

\author{
J. MAS̆EK ${ }^{a}$, I. TuREK ${ }^{b, c}$, J. KudrnovskÝ ${ }^{a}$, F. MÁCA ${ }^{a}$ \\ AND V. DRCHAL ${ }^{a}$ \\ ${ }^{a}$ Institute of Physics, AS CR, Na Slovance 2, 18221 Prague 8, Czech Republic \\ ${ }^{b}$ Institute of Physics of Materials, AS CR \\ Žižkova 22, 61662 Brno, Czech Republic \\ ${ }^{c}$ Faculty of Mathematics and Physics, Charles University \\ Ke Karlovu 5, 12116 Prague, Czech Republic
}

\begin{abstract}
We use the density-functional theory to calculate the total energy of mixed crystals $(\mathrm{Ga}, \mathrm{Mn})$ As with a small concentration of various donors. We find that the formation energy of Mn depends strongly on the partial concentrations of $\mathrm{Mn}$ in the substitutional and interstitial positions, and on the concentration of other dopants. The composition dependence of the formation energies represents an effective feedback mechanism, resulting in the self-compensation property of $(\mathrm{Ga}, \mathrm{Mn})$ As. We show that the partial concentrations of both substitutional and interstitial Mn increase proportionally to the total concentration of Mn.
\end{abstract}

PACS numbers: 71.15.Ap, 71.20.Nr, 71.55.Eq, 75.50.Pp

\section{Introduction}

The dilute magnetic semiconductors (DMS), such as GaAs doped with a large amount of Mn, represent an important class of mixed crystals with promising applications in spin electronics [1]. The ferromagnetic behavior of these materials is mediated by the holes in the valence band $[2,3]$. It is sensitive to the number of free carriers and to the level of charge compensation. Mn atoms substituted in the cation sublattice of a III-V semiconductor are acceptors and produce one hole 
each. It is known, however, that some Mn atoms occupy the interstitial positions and act as double donors [4-8].

The interplay between the substitutional and interstitial incorporation of $\mathrm{Mn}$ into the GaAs lattice has, together with co-doping (cf. Ref. [9]), a crucial effect on the physical properties of the mixed crystal (Ga,Mn)As. A systematic study of formation energies of the substitutional $\left(\mathrm{Mn}_{\mathrm{Ga}}\right)$ and interstitial $\left(\mathrm{Mn}_{\mathrm{int}}\right)$ manganese can help to understand it on a microscopic level.

In the case of weak doping small changes in the impurity concentration can easily move the Fermi energy $E_{\mathrm{F}}$ across the band gap with a negligible influence on the density of states. That is why the dependence of the formation energies on the number of electrically active impurities is usually represented by their dependence on $E_{\mathrm{F}}$. The Fermi-level dependent formation energy is obtained by adding (or subtracting) $\Delta E_{\mathrm{F}}$ to the formation energy calculated for a particular electronic configuration [10]. In the case of a strongly doped and mixed crystals, however, the redistribution of the electron states in the valence band due to the impurities cannot be neglected and the density-of-states effect modifies the simple Fermi-level rule for the formation energies.

That is why the knowledge of the formation energy as a function of the impurity concentrations is necessary in the DMS. To calculate the energy needed to incorporate $\mathrm{Mn}$ and other impurities in a mixed crystal, we use the trick relating them to the composition-dependent total energy of the mixed crystal [11]. This quantity is obtained within the density-functional theory for a series of ( $\mathrm{Ga}, \mathrm{Mn}) \mathrm{As}$ mixed crystals with various content of $\mathrm{Mn}$ in substitutional and interstitial positions, and with a variable concentration of the compensating donors. The use of the coherent potential approximation (CPA) combined with the tight-binding linearized-muffin-tin-orbital method (TB-LMTO) [12] makes possible to change the chemical composition continuously. The lattice relaxation around the impurities and the clustering of the Mn atoms are omitted within the CPA. For simplicity, we consider only the interstitial $\mathrm{Mn}$ atoms in the $\mathrm{T}\left(\mathrm{As}_{4}\right)$ position; the energy of the other $\mathrm{T}\left(\mathrm{Ga}_{4}\right)$ position is almost the same [13]. The formation energies are obtained as the first derivatives of the total energy with respect to the corresponding partial concentration [11].

Assuming a quasi-equilibrium deposition conditions, characterized by an effective growth temperature, we use the calculated formation energies to estimate the numbers of $\mathrm{Mn}_{\mathrm{Ga}}$ and $\mathrm{Mn}_{\mathrm{int}}$ in (Ga,Mn)As mixed crystal. We also present a simple way to determine the partial concentrations directly from the composition dependence of the formation energies, without solving thermodynamical balance equations.

\section{Composition dependence of the formation energies}

We consider an impure or mixed crystal with several kinds of impurities $I_{1}, I_{2}$, etc. The total energy of the mixed crystal $W\left(x_{1}, x_{2}, ..\right)$, normalized to a unit 
cell, depends on their molar concentration $x_{i}$. As we showed recently [11], the formation energy $E_{i}$ of an impurity $I_{i}$ can be obtained by differentiating $W\left(x_{1}, x_{2}, ..\right)$ with respect to $x_{i}$, namely

$$
E_{i}\left(x_{1}, x_{2}, . .\right)=\frac{\partial W\left(x_{1}, x_{2}, . .\right)}{\partial x_{i}}-E^{\text {atom }}\left(I_{i}\right)+E^{\text {atom }}(\text { host }) .
$$

The last two terms in Eq. (1) are the total energies of a free-standing atom $I_{i}$ and of the corresponding atom of the host, which has been replaced by $I_{i}$.

Generally, the definition of the formation energy is not unique and depends on the way in which the atomic energies $E^{\text {atom }}$ (host) and $E^{\text {atom }}\left(I_{i}\right)$ in Eq. (1) are obtained. We use the energies of neutral atoms in their ground state. It is important to notice, however, that the additional constant in Eq. (1) does not depend on the actual chemical composition of the material. It is not important for the concentration-dependent trends we have in mind.

That is why we consider now only the relative formation energies $\Delta E_{i}$, obtained from their actual values $E_{i}$ by subtracting the corresponding formation energy calculated for the reference material. As a reference, we take $\mathrm{Ga}_{0.96} \mathrm{Mn}_{0.04} \mathrm{As}$ with all $\mathrm{Mn}$ atoms in regular $\mathrm{Mn}_{\mathrm{G} a}$ positions.

The composition dependence of the formation energies $E_{i}$ is characterized by the coefficients of the linear expansion around the reference point,

$$
K_{i j} \equiv \frac{\partial E_{i}}{\partial x_{j}}=\frac{\partial^{2} W\left(x_{1}, x_{2}, . .\right)}{\partial x_{i} \partial x_{j}} .
$$

The correlation energies $K_{i j}$ [11] form a symmetric matrix. $K_{i j}<0$ means that the presence of the defects $I_{i}$ supports formation of $I_{j}$ and vice versa; $K_{i j}>0$ indicates the opposite tendency.

The dependence of the relative formation energy $\Delta E$ of $\mathrm{Mn}_{\mathrm{Ga}}$ on the concentration of various donors is summarized in Fig. 1. We considered four representative examples. $\mathrm{Se}_{\mathrm{As}}$ and $\mathrm{Si}_{\mathrm{Ga}}$ are typical donors with one extra electron, situated at anion and cation sublattice, respectively. The other two cases, i.e., As antisite defect $\mathrm{As}_{\mathrm{Ga}}$ and $\mathrm{Mn}_{\text {int }}$ are the most important native defects in ( $\left.\mathrm{Ga}, \mathrm{Mn}\right) \mathrm{As}$, both acting as double donors. Figure 1 shows that the formation energy of $\mathrm{Mn}_{\mathrm{Ga}}$ decreases in the presence of an increasing number of donors. The curves are grouped into pairs according to the charge state of the donors, with only a minor influence of the particular chemical origin of the defect. The dependence is almost linear for low concentrations and the slope of the function is roughly proportional to the charge state of the donor. This all indicates that the variations of the formation energy of $\mathrm{Mn}_{\mathrm{Ga}}$ are mostly determined by the Fermi-level effect, not by the redistribution of the density of states induced by the other defects.

Analogous results are obtained for the formation energy of the interstitial Mn in the $\mathrm{T}\left(\mathrm{As}_{4}\right)$ position, as shown in Fig. 2. In this case, however, the formation energy of $\mathrm{Mn}_{\mathrm{int}}$ increases with increasing number of the donors. It is important to notice that the steep increase in the formation energy represents a feedback 


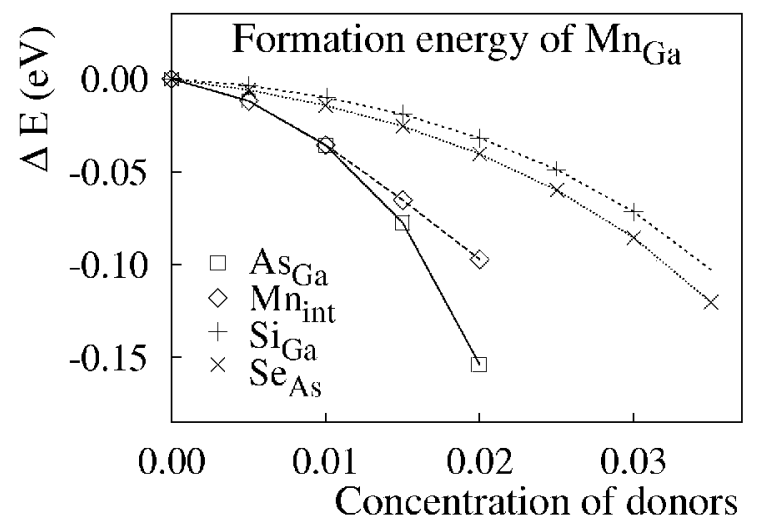

Fig. 1. Relative formation energy $\Delta E$ of the substitutional $\mathrm{Mn}_{\mathrm{Ga}}$ in $\mathrm{Ga}_{0.96} \mathrm{Mn}_{0.04} \mathrm{As}$ as a function of the concentration of various donors.

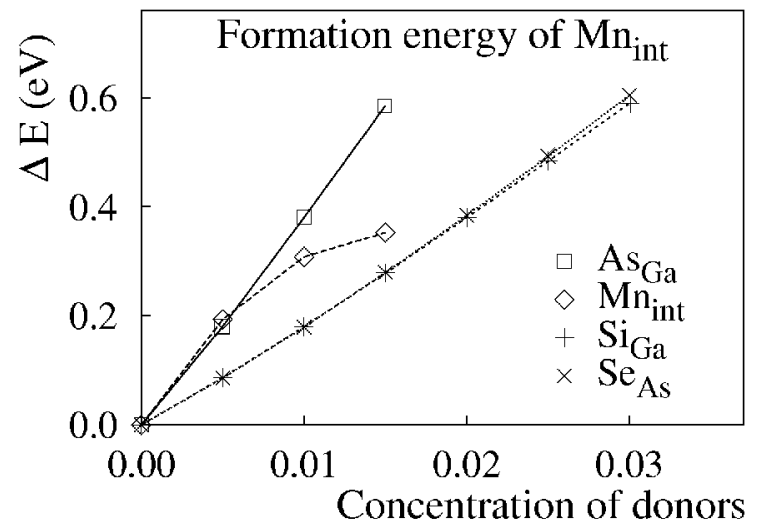

Fig. 2. Relative formation energy $\Delta E$ of the interstitial $\mathrm{Mn}$ in $\mathrm{Ga}_{0.96} \mathrm{Mn}_{0.04} \mathrm{As}$ as a function of the concentration of various donors.

mechanism limiting efficiently the number of $\mathrm{Mn}_{\text {int }}$. The same is valid also for the formation energy of the As antisite defect.

Figure 3 shows the formation energies of the two native defects, i.e. AsGa and $\mathrm{Mn}_{\mathrm{int}}$, in the mixed crystal with varying number of the substitutional $\mathrm{Mn}$ in the Ga sublattice. Notice that both relative quantities, being pinned to zero for the reference material with $4 \%$ of $\mathrm{Mn}_{\mathrm{Ga}}$, are almost identical. In both cases, the formation energy is a decreasing function, indicating an increasing probability of formation of these defects in materials with a higher concentration of Mn.

This self-compensation tendency is a very important mechanism controlling the basic physical properties of $(\mathrm{Ga}, \mathrm{Mn}) \mathrm{As}$ mixed crystals. It is the reason for the observed low doping efficiency of $\mathrm{Mn}$ in GaAs [14]. The increasing number of both $\mathrm{As}_{\mathrm{Ga}}$ and $\mathrm{Mn}_{\text {int }}$ also explains the expansion of the lattice of (Ga,Mn)As with an increasing concentration of $\mathrm{Mn}$ [15]. 


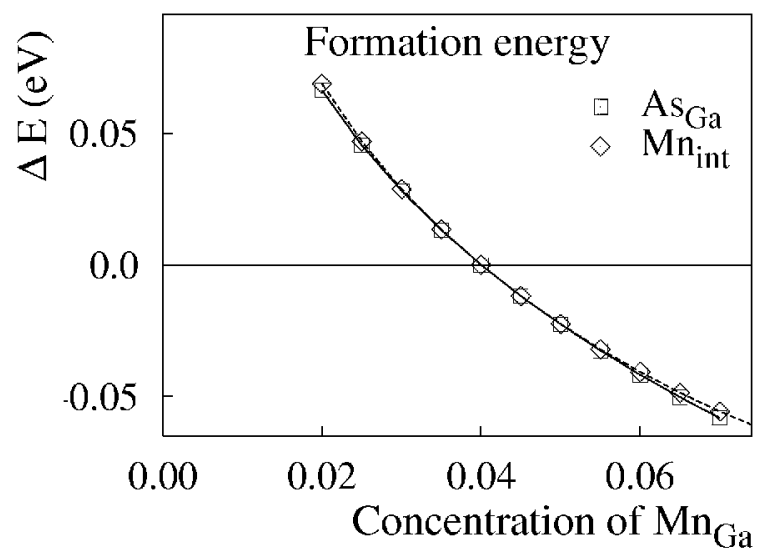

Fig. 3. The formation energy of the interstitial $\mathrm{Mn}$ and As antisite defect in $\mathrm{Ga}_{1-x} \mathrm{Mn}_{x}$ As as a function of the concentration $x$ of $\mathrm{Mn}$ atoms substituted in the Ga sublattice. The formation energies are referred to the values corresponding to $\mathrm{Ga}_{0.96} \mathrm{Mn}_{0.04} \mathrm{As}$.

\section{Dynamical equilibrium between $\mathbf{M n}_{\mathrm{Ga}}$ and $\mathbf{M n}_{\mathrm{int}}$}

In this Section, we use the calculated formation energies to simulate the incorporation of $\mathrm{Mn}$ into the (Ga,Mn)As mixed crystal. We assume that the probabilities that an $\mathrm{Mn}$ atom occupies either substitutional or interstitial position are related to the corresponding formation energies $E_{S}$ and $E_{I}$ also in the non-equilibrium epitaxial growth. As a simplest approximation, we characterize the deposition condition with some effective temperature $T_{\text {eff }}$ and use the corresponding Boltzmann weighting factors.

To this purpose, the absolute formation energies of $\mathrm{Mn}$ in the two crystallographic positions, i.e. $\mathrm{Mn}_{\mathrm{Ga}}$ and $\mathrm{Mn}_{\mathrm{int}}$, are required. They are obtained for the reference system $\mathrm{Ga}_{0.96} \mathrm{Mn}_{0.04} \mathrm{As}$ including the additive terms from Eq. (1). For the epitaxial growth, it is reasonable to use $E^{\text {atom }}$ calculated for isolated atoms as stated above. A linear interpolation for the dependence of $E_{S}$ and $E_{I}$ on the corresponding partial concentrations $x_{S}$ and $x_{I}$ is used

$$
\begin{aligned}
& E_{S}\left(x_{S}, x_{I}\right)=E_{S}^{0}+\kappa_{S S} x_{S}+\kappa_{S I} x_{I}, \\
& E_{I}\left(x_{S}, x_{I}\right)=E_{I}^{0}+\kappa_{I S} x_{S}+\kappa_{I I} x_{I}
\end{aligned}
$$

with $E_{S}^{0}=0.31 \mathrm{eV}, E_{I}^{0}=0.42 \mathrm{eV}, \kappa_{S S}=-0.17 \mathrm{eV}, \kappa_{S I}=\kappa_{I S}=-6.03 \mathrm{eV}$, and $\kappa_{I I}=10.33 \mathrm{eV}$.

The composition dependent formation energies, Eqs. (3, 4), define the thermodynamic probabilities $p_{S}$ and $p_{I}$ that extra Mn atoms occupy substitutional or interstitial position in the mixed crystal with a given composition. They are

$$
p_{S, I}=\frac{\exp \left(-E_{S, I} / k T_{\mathrm{eff}}\right)}{\exp \left(-E_{S} / k T_{\mathrm{eff}}\right)+\exp \left(-E_{I} / k T_{\mathrm{eff}}\right)} .
$$


On the other hand, these probabilities determine the number of $\mathrm{Mn}$ atoms that substitute for Ga or occupy the interstitial positions. The resulting changes of the partial concentrations $x_{S}$ and $x_{I}$ due to the variation $\mathrm{d} x$ of the total concentration $x$ of $\mathrm{Mn}$ are as follows:

$$
\mathrm{d} x_{S}=p_{S} \mathrm{~d} x, \mathrm{~d} x_{I}=p_{I} \mathrm{~d} x,
$$

and the dependence of $x_{S}$ and $x_{I}$ on $x$ can be obtained by the integration of Eq. (6).

Figure 4 shows the solution of Eq. (6) for $T_{\text {eff }}=500 \mathrm{~K}$. For the lowest concentrations $(x<0.015), \mathrm{Mn}$ atoms occupy preferentially the substitutional positions which have a lower formation energy (cf. Eqs. $(3,4)$ ). For a higher concentration of Mn, however, the difference of $E_{I}\left(x_{S}, x_{I}\right)-E_{S}\left(x_{S}, x_{I}\right)$ decreases and approaches zero. From this point, both positions can be occupied with a comparable probability, and the partial concentrations of both $\mathrm{Mn}_{\mathrm{Ga}}$ and $\mathrm{Mn}_{\text {int }}$ increase proportionally to $x$. Any deviation from the situation with $E_{S}=E_{I}$ changes the formation energies of $\mathrm{Mn}_{\mathrm{Ga}}$ and $\mathrm{Mn}_{\text {int }}$ in such a way that the dynamical equilibrium is restored. As a result, the high-concentration regime with co-existing $\mathrm{Mn}_{\mathrm{Ga}}$ and $\mathrm{Mn}_{\text {int }}$ is stabilized. This finding does not depend much on $T_{\text {eff }}$ over a wide temperature range. We can conclude that the partial concentrations of Mn are simply given by the following equation:

$$
E_{S}\left(x_{S}, x_{I}\right)=E_{I}\left(x_{S}, x_{I}\right),
$$

together with the condition $x_{S}+x_{I}=x$. Combining Eqs. $(3,4)$ with (7) we find that the proportion of $\mathrm{Mn}_{\mathrm{Ga}}$ and $\mathrm{Mn}_{\text {int }}$ atoms is roughly $3: 1$, in a very goodagreement with both experiment [4] and theoretical expectations [6, 15] for the as-grown materials.

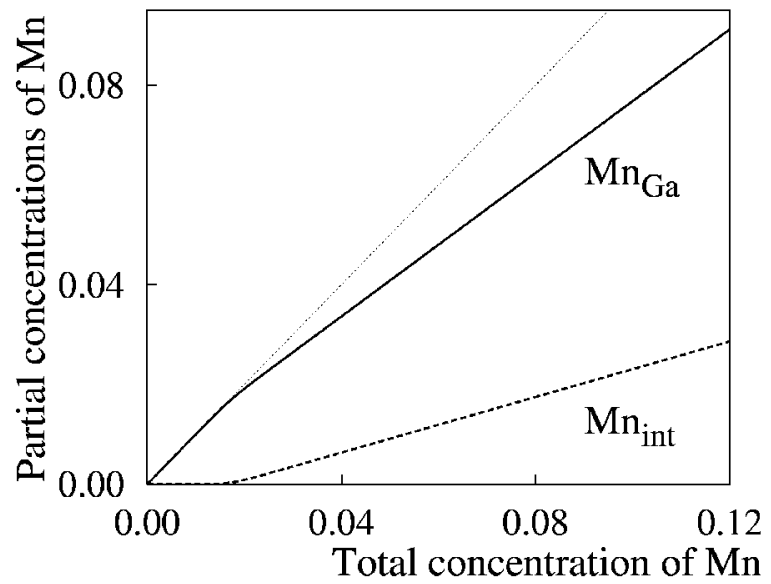

Fig. 4. The partial concentrations of $\mathrm{Mn}_{\mathrm{Ga}}$ (solid) and $\mathrm{Mn}_{\text {int }}$ (dashed) as a function of the total concentration of $\mathrm{Mn}$ in $(\mathrm{Ga}, \mathrm{Mn}) \mathrm{As}$ for $T_{\text {eff }}=500 \mathrm{~K}$. The influence of other dopants is not considered. 
It is important to point out that according to Eqs. $(3,4,7)$ both $\mathrm{Mn}_{\mathrm{Ga}}$ and $\mathrm{Mn}_{\text {int }}$ atoms remain metastable with the activation energy $\approx 0.3 \mathrm{eV}$ in the whole concentration range shown in Fig. 4. The formation energies used in our dynamical-equilibrium approach control the preferential incorporation of $\mathrm{Mn}$ atoms during the growth. The annealing process, on the other hand, depends on the barriers preventing the $\mathrm{Mn}$ atoms to leave their metastable positions. The barriers are lower for $\mathrm{Mn}_{\text {int }}$ than for $\mathrm{Mn}_{\mathrm{Ga}}$ position [16], so that the post-growth treatment can substantially reduce the number of the interstitial Mn atoms without a remarkable change of the number of $\mathrm{Mn}_{\mathrm{Ga}}$.

\section{Summary}

We have shown that the formation energies of Mn in either substitutional or interstitial position depend strongly on the partial concentrations of both $\mathrm{Mn}_{\mathrm{Ga}}$ and $\mathrm{Mn}_{\text {int }}$, and also on the number of compensating donors. Also the formation energy of $\mathrm{As}_{\mathrm{Ga}}$ antisite, the main native defect in $(\mathrm{Ga}, \mathrm{Mn}) \mathrm{As}$, is very sensitive to the concentration of $\mathrm{Mn}$.

The composition dependence of the formation energies represents a feedback mechanism which defines a dynamical equilibrium between $\mathrm{Mn}_{\mathrm{Ga}}, \mathrm{Mn}_{\text {int }}$, and other defects and impurities during the growth. In particular, we found that at higher $\mathrm{Mn}$ concentrations the number of both $\mathrm{Mn}_{\mathrm{Ga}}$ and $\mathrm{Mn}_{\text {int }}$ increases proportionally to the total concentration of $\mathrm{Mn}$ in the as-grown ( $\mathrm{Ga}, \mathrm{Mn}) \mathrm{As}$ mixed crystal.

In addition, the concentration dependence of the formation energy of the $A s_{G a}$ antisite defects indicates that an increasing number of these donors also participate in the compensation of the regular $\mathrm{Mn}_{\mathrm{Ga}}$ acceptors for higher $\mathrm{Mn}$ concentrations.

\section{Acknowledgment}

This work has been done within the project AVOZ1-010-914 of the AS CR. The financial support was provided by the Academy of Sciences of the Czech Republic (Grant No. A1010214) and by the Grant Agency of the Czech Republic $(202 / 04 / 583)$.

\section{References}

[1] H. Ohno, J. Cryst. Growth 251, 285 (2003).

[2] T. Dietl, A. Haury, Y. Merle, Phys. Rev. B 55, R 3347 (1997).

[3] T. Dietl, H. Ohno, F. Matsukura, J. Cibert, D. Ferrand, Science 287, 1019 (2000).

[4] K.M. Yu, W. Walukiewicz, T. Wojtowicz, I. Kuryliszyn, X. Liu, Y. Sasaki, J.K. Furdyna, Phys. Rev. B 65, R 201303 (2002). 
[5] J. Mašek, F. Máca, Acta Phys. Pol. A 100, 319 (2001).

[6] F. Máca, J. Mašek, Phys. Rev. B 65, 235209 (2002).

[7] S. Sanvito, G. Theurich, N. Hill, J. Supercond. 15, 85 (2002).

[8] J. Blinowski, P. Kacman, Phys. Rev. B 67, 121204 (2003).

[9] K.M. Yu, W. Walukiewicz, T. Wojtowicz, W.L. Lim, X. Liu, U. Bindley, M. Dobrowolska, J.K. Furdyna, Phys. Rev. B 68, R41308 (2003).

[10] P. Mahadevan, A. Zunger, Phys. Rev. B 68, 075202 (2003).

[11] J. Mašek, I. Turek, V. Drchal, J. Kudrnovský, F. Máca, Acta Phys. Pol. A 102, $673(2002)$.

[12] I. Turek, V. Drchal, J. Kudrnovský, M. Šob, P. Weinberger, Electronic Structure of Disordered Alloys, Surfaces and Interfaces, Kluwer, Boston 1997.

[13] J. Mašek, F. Máca, Phys. Rev. B 69, 165212 (2004).

[14] B. Beschoten, P.A. Crowell, I. Malajovich, D.D. Awschalom, F. Matsukura, A. Shen, H. Ohno, Phys. Rev. Lett. 83, 3073 (1999).

[15] J. Mašek, J. Kudrnovský, F. Máca, Phys. Rev. B 67, 153203 (2003).

[16] S.C. Erwin, A.G. Petukhov, Phys. Rev. Lett. 89, 227201 (2002). 